\title{
Algebraic methods for the solution of linear functional equations
}

\author{
Cs. Vincze, A. Varga and G. Kiss
}

July 21, 2014

\begin{abstract}
Equation

$$
\sum_{i=0}^{n} a_{i} f\left(b_{i} x+\left(1-b_{i}\right) y\right)=0
$$

belongs to the class of linear functional equations. The solutions form a linear space with respect to the usual pointwise operations. According to the classical results of the theory they must be generalized polynomials. New investigations have been started few years ago. They clarified that the existence of non-trivial solutions depends on the algebraic properties of some related families of parameters. The problem is to find the necessary and sufficient conditions for the existence of non-trivial solutions in terms of these kinds of properties. One of the most earlier results is due to Z. Daróczy [1]. It can be considered as the solution of the problem in case of $n=2$. We are going to take more steps forward by solving the problem in case of $n=3$.
\end{abstract}

\footnotetext{
${ }^{1}$ Cs. Vincze was partially supported by the European Union and the European Social Fund through the project Supercomputer, the national virtual lab (grant no.:TÁMOP4.2.2.C-11/1/KONV-2012-0010). The work is supported by the University of Debrecen's internal research project $\mathrm{RH} / 885 / 2013$.

${ }^{2}$ A. Varga was supported by the European Union co-financed by the European Social Fund. The work was carried out as a part of the TÁMOP-4.1.1.C-12/1/KONV-2012-0012 project in the framework of the New Hungarian Development Plan.

${ }^{3}$ G. Kiss was partially supported by the Hungarian National Foundation for Scientific Research, Grants No. K104178.
} 


\section{Introduction}

Consider functional equation

$$
\sum_{i=0}^{n} a_{i} f\left(b_{i} x+\left(1-b_{i}\right) y\right)=0 \quad(x, y \in I)
$$

where $I$ is a nonempty open real interval, $0<b_{0}<b_{1}<\ldots<b_{n}<1$ and $a_{0}, a_{1}, \ldots, a_{n}$ are given nonzero real numbers, such that $\sum_{i=0}^{n} a_{i}=0$ (it is natural because of the substitution $x=y$ ). The solutions form a linear space with respect to the usual pointwise operations. According to the classical results of the theory they must be generalized polynomials. New investigations have been started few years ago. They clarified that the existence of nontrivial solutions depends on the algebraic properties of some related families of parameters. We present the basic results of the theory together with some sufficient and necessary conditions for the existence of non-trivial solutions in special cases. The starting point of the investigations is summarized in the following theorem.

Theorem 1.1. [4] Let $n \geq 2$ be a given natural number. The function $f: I \rightarrow \mathbb{R}$ is a solution of equation (1) if and only if there exist symmetric $k$-additive functions $A_{k}: \mathbb{R}^{k} \rightarrow \mathbb{R}$ and $A_{0} \in \mathbb{R}$ such that

$$
f(x)=\sum_{k=1}^{n-1} A_{k}(x, \ldots, x)+A_{0} \quad(x \in I)
$$

and for any $k=1, \ldots, n-1$

$$
\left(A_{k}\right):\left\{\begin{array}{cc}
A_{k}(s, \ldots, s, t)+\sum_{i=1}^{n-1} \alpha_{i} A_{k}\left(s, \ldots, s, t \beta_{i}\right) & =0 \\
A_{k}(s, \ldots, s, t, t)+\sum_{i=1}^{n-1} \alpha_{i} A_{k}\left(s, \ldots, s, t \beta_{i}, t \beta_{i}\right) & =0 \\
\ldots & \\
\ldots & \\
\ldots & \\
A_{k}(t, \ldots, t)+\sum_{i=1}^{n-1} \alpha_{i} A_{k}\left(t \beta_{i}, \ldots, t \beta_{i}\right) & 0
\end{array}\right.
$$

where $s, t \in \mathbb{R}$,

$$
\alpha_{i}:=\frac{a_{i}}{a_{n}} \text { and } \beta_{i}:=\frac{b_{i}-b_{0}}{b_{n}-b_{0}} \quad(i=1, \ldots, n-1) .
$$


By the help of an extension theorem due to Zs. Páles [5] the proof goes back to L. Székelyhidi's basic results [7]. The system $\left(A_{k}\right)$ gives sufficient and necessary conditions for the existence of solutions of degree $1,2, \ldots$, $n-1$, respectively. The finer structure of the solutions has been investigated only the past couple of years. A. Varga and Cs. Vincze [4] applied some field homomorphisms to construct non-trivial solutions. The method leads naturally to the problem of algebraic characterization [3]. The basic results can be formulated in the following way.

Definition 1.2. $\vec{\beta}:=\left(\beta_{1}, \ldots, \beta_{m}\right) \in \mathbb{R}^{m}$ or $\mathbb{C}^{m}$ is called an algebraically dependent system if there exists a not identically zero $p \in \mathbb{Q}\left[x_{1}, \ldots, x_{m}\right]$ such that $p(\vec{\beta})=0$. Otherwise we say that it is an algebraically independent system.

The ideal

$$
\mathcal{I}(\vec{\beta}):=\left\{p \in \mathbb{Q}\left[x_{1}, \ldots, x_{m}\right] \mid p(\vec{\beta})=0\right\}
$$

of the polynomial ring $\mathbb{Q}\left[x_{1}, \ldots, x_{m}\right]$ is called the defining ideal of $\vec{\beta}$. If $\mathcal{I}(\vec{\beta})=\mathcal{I}(\vec{\gamma})$ then we say that $\vec{\beta}$ and $\vec{\gamma}$ are algebraically conjugated.

Theorem 1.3. [4] There exists a field isomorphism

$$
\delta: \mathbb{Q}\left(\beta_{1}, \ldots, \beta_{m}\right) \rightarrow \mathbb{Q}\left(\gamma_{1}, \ldots, \gamma_{m}\right)
$$

such that $\delta\left(\beta_{i}\right)=\gamma_{i}(i=1,2, \ldots, m)$ if and only if $\vec{\beta}$ and $\vec{\gamma}$ are algebraically conjugated.

Theorem 1.4. [3] Let $n \geq 2$ be a given natural number. Suppose that the outer parameters $\alpha_{i}$ 's $(i=1, \ldots, n-1)$ form an algebraically independent system. There exists a not identically zero $A_{1}: \mathbb{R} \rightarrow \mathbb{R}$ additive solution of equation

$$
\left(A_{1}\right): \quad A_{1}(t)+\sum_{i=1}^{n-1} \alpha_{i} A_{1}\left(t \beta_{i}\right)=0 \quad(t \in \mathbb{R})
$$

iff at least one of the inner parameters $\beta_{i}$ 's is transcendent.

The proof goes back to the construction of a field homomorphism $\delta_{1}$ satisfying

$$
1+\sum_{i=1}^{n-1} \alpha_{i} \delta_{1}\left(\beta_{i}\right)=0 .
$$

Note that the role of the outer and the inner parameters in Theorem 1.4 is symmetric; for the details see [3]. Another interesting pure case when all 
the inner (or the outer) parameters are algebraic numbers over the rationals. Then we have an eliminating technic due to A. Varga [2] to solve equation (3). The mixed cases (algebraically dependent systems of parameters containing transcendental numbers) are open problems.

An important recent trend in the theory of linear functional equations is the applications of spectral analysis and spectral synthesis in some related varieties. M. Laczkovich [11], A. Varga and G. Kiss [9] clarified that the existence of injective homomorphisms satisfying some special relationships is a necessary and sufficient condition for the existence of a non-trivial $k$-additive solution. The proof uses the theorem due to M. Laczkovich and G. Székelyhidi [8]. They proved that spectral analysis holds on a discrete Abelian group $G$ if the cardinality of $G$ is less than $2^{\omega}$ (the cardinality of continuum). This means that there exist non-trivial exponentionals in the varieties of the linear space of complex valued functions defined on $G$. In what follows we apply this theorem to the variety of the monomial solutions $A_{k}$ restricted to the Cartesian product $G:=\left(K^{*}\right)^{k}$, where $K^{*}:=\mathbb{Q}\left(\beta_{1}, \ldots, \beta_{n-1}\right) \backslash\{0\}$ is the multiplicative group for any $k=1, \ldots, n-1$. Suppose that $A_{k}$ is a not identically zero $k$-additive symmetric function satisfying the system $\left(A_{k}\right)$ and let the variable $t$ be fixed for a moment. Consider the left hand sides of the equations as the diagonals of the symmetric multi-additive functions

$$
\begin{gathered}
B_{k-1}\left(s_{1}, \ldots, s_{k-1}\right):=A_{k}\left(s_{1}, \ldots, s_{k-1}, t\right)+\sum_{i=1}^{n-1} \alpha_{i} A_{k}\left(s_{1}, \ldots, s_{k-1}, t \beta_{i}\right), \\
B_{k-2}\left(s_{1}, \ldots, s_{k-2}\right):=A_{k}\left(s_{1}, \ldots, s_{k-2}, t, t\right)+\sum_{i=1}^{n-1} \alpha_{i} A_{k}\left(s_{1}, \ldots, s_{k-2}, t \beta_{i}, t \beta_{i}\right), \\
\ldots \\
B_{1}\left(s_{1}\right):=A_{k}\left(s_{1}, t, \ldots, t\right)+\sum_{i=1}^{n-1} \alpha_{i} A_{k}\left(s_{1}, t \beta_{i}, \ldots, t \beta_{i}\right),
\end{gathered}
$$

respectively. It is known that the values of the diagonals determine the symmetric multi-additive functions and we have the vanishing of $B_{k-1}, \ldots$, $B_{1}$. We can set free the variable $t$ in a similar way. Therefore $\left(A_{k}\right)$ can be 
written into the multi-variable form

$$
\left(M_{k}\right):\left\{\begin{array}{cc}
A_{k}\left(s_{1}, \ldots, s_{k-1}, t_{1}\right)+\sum_{i=1}^{n-1} \alpha_{i} A_{k}\left(s_{1}, \ldots, s_{k-1}, t_{1} \beta_{i}\right) & =0, \\
A_{k}\left(s_{1}, \ldots, s_{k-2}, t_{1}, t_{2}\right)+\sum_{i=1}^{n-1} \alpha_{i} A_{k}\left(s_{1}, \ldots, s_{k-2}, t_{1} \beta_{i}, t_{2} \beta_{i}\right) & =0, \\
\ldots & \\
\ldots & \\
\ldots & \\
A_{k}\left(t_{1}, \ldots, t_{k}\right)+\sum_{i=1}^{n-1} \alpha_{i} A_{k}\left(t_{1} \beta_{i}, \ldots, t_{k} \beta_{i}\right) & 0 .
\end{array}\right.
$$

The vector space

$$
\begin{array}{r}
V:=\left\{\left.A_{k}\right|_{G} \mid A_{k}: \mathbb{R}^{k} \rightarrow \mathbb{C} \text { is k-additive satisfying the system }\left(M_{k}\right)\right. \\
\text { for any permutations of the variables in each equation }\}
\end{array}
$$

- is nontrivial: if $A_{k}$ is a non-trivial monomial solution of equation (1) then the translation invariance of the space of the solutions allows us to translate $A_{k}$ in such a way that $\left.A_{k}\right|_{G}$ is not identically zero.

- is translation invariant ${ }^{1}$ : if $\varphi \in V$ then for any $k$-tuple $\vec{x}=\left(x_{1}, \ldots, x_{k}\right)$ the map $\tau_{\vec{x}} \varphi\left(y_{1}, \ldots, y_{k}\right):=\varphi\left(x_{1} y_{1}, \ldots, x_{k} y_{k}\right)$ is in $V$.

- $\mathrm{V}$ is closed under the pointwise convergence.

Therefore $V$ is a variety on the group $G$. According to [8] spectral analysis holds on $G$ which means that there exists a not identically zero mapping $m: G \rightarrow \mathbb{C}$ in V satisfying the exponential property

$$
m\left(x_{1} y_{1}, \ldots, x_{k} y_{k}\right)=m\left(x_{1}, \ldots, x_{k}\right) m\left(y_{1}, \ldots, y_{k}\right) .
$$

We can write

$$
m\left(x_{1}, \ldots, x_{k}\right)=m\left(x_{1}, 1, \ldots, 1\right) \cdot m\left(1, x_{2}, 1, \ldots, 1\right) \cdot \ldots \cdot m\left(1, \ldots, 1, x_{k}\right),
$$

where each mapping on the right hand side can be considered as an injective homomorphism ${ }^{2}$ of $\mathbb{Q}\left(\beta_{1}, \ldots, \beta_{n-1}\right)$ into the set of complex numbers:

$$
\delta_{1}\left(x_{1}\right):=m\left(x_{1}, 1, \ldots, 1\right), \ldots, \delta_{k}\left(x_{k}\right):=m\left(1, \ldots, 1, x_{k}\right) .
$$

\footnotetext{
${ }^{1}$ Note that $\tau_{\vec{x}} \varphi$ is not a symmetric mapping in general even if it is the translate of a symmetric element in $V$. This is the reason why to write the system of equations into the multi-variable form. The condition for any permutations of the variables in each equation provides that $V$ is closed under the usual symmetrization process. The problem disappears in case of $A_{1}$ because of the single variable.

${ }^{2}$ The exponential property shows that the vanishing of $m$ at a single non-zero element implies that $m$ must be identically zero.
} 
Substituting $m$ as a product $\delta_{1} \cdot \ldots \cdot \delta_{k}$ into the equations (together with the permutations of the variables) of $\left(M_{k}\right)$ we have the following theorem.

Theorem 1.5. [9] Let $k \in\{1, \ldots, n-1\}$ be given. There exists a not identically zero $k$-additive symmetric function $A_{k}$ such that its diagonalization is a solution of equation (1) if and only if there exists a collection of injective homomorphisms

$$
\delta_{1}, \ldots, \delta_{k}: \mathbb{Q}\left(\beta_{1}, \ldots, \beta_{n-1}\right) \rightarrow \mathbb{C}
$$

such that

$$
\left(F_{k}\right):\left\{\begin{array}{ccc}
1+\sum_{i=1}^{n-1} \alpha_{i} \delta_{j}\left(\beta_{i}\right) & = & 0, \quad(j=1, \ldots, k) \\
1+\sum_{i=1}^{n-1} \alpha_{i} \delta_{j_{1}}\left(\beta_{i}\right) \delta_{j_{2}}\left(\beta_{i}\right) & = & 0,\left(j_{1} \neq j_{2}, j_{1}, j_{2}=1, \ldots, k\right) \\
\ldots & & \\
\ldots & & \\
\ldots &
\end{array}\right.
$$

where $\alpha_{i}:=\frac{a_{i}}{a_{n}}$ and $\beta_{i}:=\frac{b_{i}-b_{0}}{b_{n}-b_{0}} \quad(i=1, \ldots, n-1)$.

To see that the condition is sufficient let us define $A_{k}$ as

$$
A_{k}:=\sum_{\sigma} \delta_{\sigma(1)} \cdot \ldots \cdot \delta_{\sigma(k)}
$$

where $\sigma$ runs through the permutations of $1,2, \ldots, k$ (it is a usual symmetrization process). The real solutions are the real and the imaginary parts of $A_{k}$, respectively. As a recent result M. Laczkovich and G. Kiss [10] proved that spectral synthesis also holds in the variety $V$ and the space of the restricted solutions $\left.A_{k}\right|_{G}$ is spanned by exponential monomials - they also show that exponential monomials can be written in terms of exponentials and differential operators on the field $\mathbb{Q}\left(\beta_{1}, \ldots, \beta_{n-1}\right)$.

The basic problem is when injective homomorphisms satisfying $\left(F_{k}\right)$ exist. It is natural to formulate conditions in terms of the algebraic properties of the parameters. The aim is to find them in some special cases. Z. Daróczy [1] proved, that there exists a not identically zero $A_{1}: \mathbb{R} \rightarrow \mathbb{R}$ additive function satisfying equation $\left(A_{1}\right)$ in case of $n=2$ if and only if the reals $\beta_{1}$ and $-\left(1 / \alpha_{1}\right)$ are algebraically conjugated (i.e. they are transcendent or they are 
algebraic with the same defining polynomial). This condition is equivalent to the existence of an injective homomorphism $\delta_{1}$ satisfying

$$
\delta_{1}\left(\beta_{1}\right)=-\left(1 / \alpha_{1}\right) \Leftrightarrow 1+\alpha_{1} \delta_{1}\left(\beta_{1}\right)=0 .
$$

As we can see the value of $\delta_{1}\left(\beta_{1}\right)$ can be directly expressed from $\left(F_{1}\right)$ in case of $n=2$. In case of $n>2$ the situation is more complicated ${ }^{3}$.

In what follows we are going to present the full solution for the first nontrivial case of $n=3$ with several parameters. We formulate necessary and sufficient conditions for the existence of non-trivial first and second order solutions of equation (1).

\section{The case of $n=3$}

In case of $n=3$ the maximal degree of the solutions of equation (1) is 2. Because of Theorem 1.5 there exists a not identically zero symmetric biadditive function such that its diagonalization is a solution of equation

$$
\sum_{i=0}^{3} a_{i} f\left(b_{i} x+\left(1-b_{i}\right) y\right)=0 \quad(x, y \in I)
$$

iff there exist injective homomorphisms $\delta_{1}, \delta_{2}: \mathbb{Q}\left(\beta_{1}, \beta_{2}\right) \rightarrow \mathbb{C}$ such that

$$
\left(F_{2}\right):\left\{\begin{array}{ccc}
1+\alpha_{1} \delta_{1}\left(\beta_{1}\right)+\alpha_{2} \delta_{1}\left(\beta_{2}\right) & = & 0 \\
1+\alpha_{1} \delta_{2}\left(\beta_{1}\right)+\alpha_{2} \delta_{2}\left(\beta_{2}\right) & = & 0 \\
1+\alpha_{1} \delta_{1}\left(\beta_{1}\right) \delta_{2}\left(\beta_{1}\right)+\alpha_{2} \delta_{1}\left(\beta_{2}\right) \delta_{2}\left(\beta_{2}\right) & = & 0
\end{array}\right.
$$

where

$$
\alpha_{i}:=\frac{a_{i}}{a_{3}} \text { and } \beta_{i}:=\frac{b_{i}-b_{0}}{b_{3}-b_{0}} \quad(i=1,2) .
$$

In what follows we shall frequently use that any injective homomorphism $\delta: \mathbb{Q}\left(\beta_{1}, \beta_{2}\right) \rightarrow \mathbb{C}$ can be extended to an authomorphism $\delta: \mathbb{C} \rightarrow \mathbb{C}$.

\footnotetext{
${ }^{3}$ In some sense Theorem 1.4 can be considered as one of the generalizations of Daróczy's original result.
} 


\subsection{The existence of a nontrivial $A_{2}$ term}

Lemma 2.1. Let $\alpha_{1}, \alpha_{2}$ and $\beta_{1}, \beta_{2}$ be nonzero real numbers such that $\beta_{1}<$ $\beta_{2}$. There exist injective homomorphisms

$$
\delta_{1}, \delta_{2}: \mathbb{Q}\left(\beta_{1}, \beta_{2}\right) \rightarrow \mathbb{C}
$$

such that the system $\left(F_{2}\right)$ holds if and only if there exist $\mu \in \mathbb{C}$ satisfying

$$
(\star)\left\{\begin{array}{l}
\alpha_{1}=\delta_{2}\left(\frac{1-\beta_{2}}{\beta_{2}-\beta_{1}} \cdot \frac{\mu\left(\beta_{1}-1\right)+1}{\beta_{1}}\right), \\
\alpha_{2}=\delta_{2}\left(\frac{\beta_{1}-1}{\beta_{2}-\beta_{1}} \cdot \frac{\mu\left(\beta_{2}-1\right)+1}{\beta_{2}}\right)
\end{array}\right.
$$

and an injective homomorphism $\varphi: \mathbb{Q}\left(\beta_{1}, \beta_{2}\right) \rightarrow \mathbb{C}$ such that

$$
(\star \star) \quad\left\{\begin{array}{l}
\varphi\left(\beta_{1}\right)=\frac{\beta_{1}}{\mu\left(\beta_{1}-1\right)+1} \\
\varphi\left(\beta_{2}\right)=\frac{\beta_{2}}{\mu\left(\beta_{2}-1\right)+1}
\end{array}\right.
$$

Proof. $\Leftarrow$ It is easy to check, that if $(\star)$ and $(\star \star)$ hold, then the system $\left(F_{2}\right)$ is satisfied under the choice $\delta_{1}:=\delta_{2} \circ \varphi$.

$\Rightarrow$ Using the standard Cramer-rule for the first and third equations of the system $\left(F_{2}\right)$ we get that

$$
\alpha_{1}=\frac{1-\delta_{2}\left(\beta_{2}\right)}{\delta_{1}\left(\beta_{1}\right)\left(\delta_{2}\left(\beta_{2}\right)-\delta_{2}\left(\beta_{1}\right)\right)} \quad \text { and } \quad \alpha_{2}=\frac{\delta_{2}\left(\beta_{1}\right)-1}{\delta_{1}\left(\beta_{2}\right)\left(\delta_{2}\left(\beta_{2}\right)-\delta_{2}\left(\beta_{1}\right)\right)} .
$$

Similarly, from the second and third equations of the system we have

$$
\alpha_{1}=\frac{1-\delta_{1}\left(\beta_{2}\right)}{\delta_{2}\left(\beta_{1}\right)\left(\delta_{1}\left(\beta_{2}\right)-\delta_{1}\left(\beta_{1}\right)\right)} \quad \text { and } \quad \alpha_{2}=\frac{\delta_{1}\left(\beta_{1}\right)-1}{\delta_{2}\left(\beta_{2}\right)\left(\delta_{1}\left(\beta_{2}\right)-\delta_{1}\left(\beta_{1}\right)\right)} .
$$

Comparing (5) and (6) the basic properties of the homomorphisms imply that

$$
\delta_{1}\left(\frac{\beta_{1}\left(1-\beta_{2}\right)}{\beta_{2}-\beta_{1}}\right)=\delta_{2}\left(\frac{\beta_{1}\left(1-\beta_{2}\right)}{\beta_{2}-\beta_{1}}\right)
$$

Since $\lambda:=\frac{\beta_{1}\left(1-\beta_{2}\right)}{\beta_{2}-\beta_{1}}$ is an invariant parameter, $\delta_{1}$ and $\delta_{2}$ act on $\mathbb{Q}(\lambda)$ in the same way. If $\varphi:=\delta_{2}^{-1} \circ \delta_{1}$, then $\varphi(\lambda)=\lambda$, that is

$$
\frac{\varphi\left(\beta_{1}\right)\left(1-\varphi\left(\beta_{2}\right)\right)}{\varphi\left(\beta_{2}\right)-\varphi\left(\beta_{1}\right)}=\frac{\beta_{1}\left(1-\beta_{2}\right)}{\beta_{2}-\beta_{1}} .
$$


Moreover $\varphi(x)=x \quad(x \in \mathbb{Q}(\lambda))$. Equation (8) implies that the quadruples of the complex numbers $\beta_{1}, 1,0, \beta_{2}$ and $\varphi\left(\beta_{1}\right), 1,0, \varphi\left(\beta_{2}\right)$ have the same cross ratio $^{4}$ :

$$
\left(\beta_{1} 10 \beta_{2}\right)=\left(\varphi\left(\beta_{1}\right) 10 \varphi\left(\beta_{2}\right)\right) .
$$

It is well known, that the cross ratio is invariant under the Möbius transformation. It is also true, that the Möbius transformation group is 3-transitive, i.e. for given complex numbers $z_{i}, w_{i}(i=1,2,3)$ there exist a uniquely determined Möbius transformation

$$
M(z)=\frac{a z+b}{c z+d} \quad(z \in \mathbb{C})
$$

where $a, b, c, d \in \mathbb{C}$ and $a d-b c \neq 0$ such that $M\left(z_{i}\right)=w_{i} \quad(i=1,2,3)$.

Using (9) we get that there exists a uniquely determined Möbius transformation such that

$$
M\left(\beta_{1}\right)=\varphi\left(\beta_{1}\right), \quad M(1)=1, \quad M(0)=0, \quad M\left(\beta_{2}\right)=\varphi\left(\beta_{2}\right)
$$

$M(0)=0$ says that $b=0$. Since $M(1)=1$, we have that $a=c+d$. Therefore

$$
M(z)=\frac{z}{\mu(z-1)+1},
$$

where $\mu=\frac{c}{c+d}$. Especially

$$
\varphi\left(\beta_{1}\right)=\frac{\beta_{1}}{\mu\left(\beta_{1}-1\right)+1} \text { and } \varphi\left(\beta_{2}\right)=\frac{\beta_{2}}{\mu\left(\beta_{2}-1\right)+1} .
$$

Since $\varphi:=\delta_{2}^{-1} \circ \delta_{1}$, it follows that

$$
\delta_{1}\left(\beta_{1}\right)=\frac{\delta_{2}\left(\beta_{1}\right)}{\delta_{2}(\mu)\left(\delta_{2}\left(\beta_{1}\right)-1\right)+1} \text { and } \delta_{1}\left(\beta_{2}\right)=\frac{\delta_{2}\left(\beta_{2}\right)}{\delta_{2}(\mu)\left(\delta_{2}\left(\beta_{2}\right)-1\right)+1} .
$$

Substituting these expressions as $\delta_{1}\left(\beta_{1}\right)$ and $\delta_{1}\left(\beta_{2}\right)$ into $\left(F_{2}\right)$ we have

$$
\left(F_{2}^{\star}\right):\left\{\begin{array}{l}
1+\alpha_{1}^{\star} \delta_{2}\left(\beta_{1}\right)+\alpha_{2}^{\star} \delta_{2}\left(\beta_{2}\right)=0 \\
1+\alpha_{1} \delta_{2}\left(\beta_{1}\right)+\alpha_{2} \delta_{2}\left(\beta_{2}\right)=0 \\
1+\alpha_{1}^{\star} \delta_{2}^{2}\left(\beta_{1}\right)+\alpha_{2}^{\star} \delta_{2}^{2}\left(\beta_{2}\right)=0
\end{array}\right.
$$

\footnotetext{
${ }^{4}$ Recall that the cross ratio is real iff the elements are lying on the same line or circle.
} 
where

$$
\alpha_{1}^{\star}=\frac{\alpha_{1}}{\delta_{2}(\mu)\left(\delta_{2}\left(\beta_{1}\right)-1\right)+1} \quad \text { and } \quad \alpha_{2}^{\star}=\frac{\alpha_{2}}{\delta_{2}(\mu)\left(\delta_{2}\left(\beta_{2}\right)-1\right)+1 .}
$$

Using the Cramer-rule for the first and third equations of the system $\left(F_{2}^{\star}\right)$ we get that

$$
\alpha_{1}^{\star}=\frac{1-\delta_{2}\left(\beta_{2}\right)}{\delta_{2}\left(\beta_{1}\right)\left(\delta_{2}\left(\beta_{2}\right)-\delta_{2}\left(\beta_{1}\right)\right)} \quad \text { and } \quad \alpha_{2}^{\star}=\frac{\delta_{2}\left(\beta_{1}\right)-1}{\delta_{2}\left(\beta_{2}\right)\left(\delta_{2}\left(\beta_{2}\right)-\delta_{2}\left(\beta_{1}\right)\right)} .
$$

Comparing (10) and (11) the basic properties of the homomorphisms imply that

$$
\left\{\begin{array}{l}
\alpha_{1}=\delta_{2}\left(\frac{1-\beta_{2}}{\beta_{2}-\beta_{1}} \cdot \frac{\mu\left(\beta_{1}-1\right)+1}{\beta_{1}}\right), \\
\alpha_{2}=\delta_{2}\left(\frac{\beta_{1}-1}{\beta_{2}-\beta_{1}} \cdot \frac{\mu\left(\beta_{2}-1\right)+1}{\beta_{2}}\right) .
\end{array}\right.
$$

Combining Theorems 1.5, 1.3 and Lemma 2.1 we have the following result:

Theorem 2.2. Let the reals $a_{i} \neq 0, b_{i}(i=0,1,2,3)$ be given such that

$$
a_{0}+a_{1}+a_{2}+a_{3}=0 \text { and } 0<b_{0}<b_{1}<b_{2}<b_{3}<1 \text {. }
$$

There exist a not identically zero symmetric biadditive function such that its diagonalization is a solution of equation

$$
\sum_{i=0}^{3} a_{i} f\left(b_{i} x+\left(1-b_{i}\right) y\right)=0 \quad(x, y \in I),
$$

if and only if there exists $\mu \in \mathbb{C}$ such that

- $\left(\beta_{1}, \beta_{2}\right)$ and $\left(\frac{\beta_{1}}{\mu\left(\beta_{1}-1\right)+1}, \frac{\beta_{2}}{\mu\left(\beta_{2}-1\right)+1}\right)$ are algebraically conjugated,

- $\left(\alpha_{1}, \alpha_{2}\right)$ and $\left(\frac{1-\beta_{2}}{\beta_{2}-\beta_{1}} \cdot \frac{\mu\left(\beta_{1}-1\right)+1}{\beta_{1}}, \frac{\beta_{1}-1}{\beta_{2}-\beta_{1}} \cdot \frac{\mu\left(\beta_{2}-1\right)+1}{\beta_{2}}\right)$ are algebraically conjugated,

where $\alpha_{i}:=\frac{a_{i}}{a_{3}}$ and $\beta_{i}:=\frac{b_{i}-b_{0}}{b_{3}-b_{0}} \quad(i=1,2)$. 


\subsection{The existence of a nontrivial $A_{1}$ term}

Theorem 1.1 and 1.5 imply, that there exists a not identically zero additive solution $A_{1}$ of equation (4) iff $A_{1}$ satisfies equation

$$
A_{1}(t)+\alpha_{1} A_{1}\left(t \beta_{1}\right)+\alpha_{2} A_{1}\left(t \beta_{2}\right)=0 \quad(t \in \mathbb{R})
$$

or equivalently, there exists an injective homomorphism $\delta_{1}: \mathbb{Q}\left(\beta_{1}, \beta_{2}\right) \rightarrow \mathbb{C}$ such that

$$
\begin{gathered}
1+\alpha_{1} \delta_{1}\left(\beta_{1}\right)+\alpha_{2} \delta_{1}\left(\beta_{2}\right)=0 \\
\text { where } \alpha_{i}:=\frac{a_{i}}{a_{n}} \text { and } \beta_{i}:=\frac{b_{i}-b_{0}}{b_{n}-b_{0}} \quad(i=1,2) .
\end{gathered}
$$

Our aim is to decide the existence of not identically zero additive solutions in view of the parameters $\vec{\alpha}:=\left(\alpha_{1}, \alpha_{2}\right)$ and $\vec{\beta}:=\left(\beta_{1}, \beta_{2}\right)$. According to Theorem 1.4 and the concluding remark about the symmetric role of the parameters it is enough to investigate the case of algebraically dependent parameters. Suppose that $\vec{\beta}:=\left(\beta_{1}, \beta_{2}\right)$ is an algebraically dependent system. Then we have a not identically zero irreducible polynomial $p \in \mathcal{I}\left(\beta_{1}, \beta_{2}\right)$, i.e.

$$
p\left(\beta_{1}, \beta_{2}\right)=0 .
$$

Using the basic properties of homomorhisms we have: $p\left(\delta_{1}\left(\beta_{1}\right), \delta_{1}\left(\beta_{2}\right)\right)=0$. Equation (13) says that

$p\left(\delta_{1}\left(\beta_{1}\right),-\frac{1}{\alpha_{2}}\left(1+\alpha_{1} \delta_{1}\left(\beta_{1}\right)\right)\right)=0$ and $p\left(-\frac{1}{\alpha_{1}}\left(1+\alpha_{2} \delta_{1}\left(\beta_{2}\right)\right), \delta_{1}\left(\beta_{2}\right)\right)=0$.

Taking the polynomials

$$
p_{1}(x):=p\left(x,-\frac{1}{\alpha_{2}}\left(1+\alpha_{1} x\right)\right)=\sum_{k, l} p_{k, l} x^{k}(-1)^{l} \frac{1}{\alpha_{2}^{l}}\left(1+\alpha_{1} x\right)^{l}
$$

and

$$
\left.p_{2}(y):=p\left(-\frac{1}{\alpha_{1}}\left(1+\alpha_{2} y\right), y\right)\right)=\sum_{k, l} p_{k, l} y^{l}(-1)^{k} \frac{1}{\alpha_{1}^{k}}\left(1+\alpha_{2} y\right)^{k}
$$

we can determine $\delta_{1}\left(\beta_{1}\right)$ or $\delta_{1}\left(\beta_{2}\right)$ as one of the finitely many roots provided that none of the polynomials $p_{1}$ and $p_{2}$ are identically zero. In what follows 
we investigate the possible cases. It is easy to check that the constant terms of $p_{1}$ and $p_{2}$ can be given by the sums

$$
\sum_{k=0, l} p_{0, l}(-1)^{l} \frac{1}{\alpha_{2}^{l}} \text { and } \sum_{k, l=0} p_{k, 0}(-1)^{k} \frac{1}{\alpha_{1}^{k}}, \text { respectively. }
$$

Consider the polynomials

$$
r(t):=\sum_{k=0, l} p_{0, l}(-1)^{l} t^{l} \text { and } q(t):=\sum_{k, l=0} p_{k, 0}(-1)^{k} t^{k} .
$$

The constant terms of $p_{1}$ and $p_{2}$ are equal to $r\left(\frac{1}{\alpha_{2}}\right)$ and $q\left(\frac{1}{\alpha_{1}}\right)$, respectively.

Lemma 2.3. None of the polynomials $r$ and $q$ are identically zero.

Proof. Suppose that for example $r$ is the identically zero polynomial. Then $p_{0, l}=0$ for any indices $l$ and, consequently, we can write that $p(x, y)=$ $x \cdot \tilde{p}(x, y)$ for some $\tilde{p} \in \mathbb{Q}\left[x_{1}, x_{2}\right]$. This contradicts to the irreducibility.

To finish the discussion of the existence of a non-zero additive term in the solution of equation (4) we distinguish the following three cases:

$$
\begin{aligned}
& \text { I. } r\left(\frac{1}{\alpha_{2}}\right)=0 \text { and } q\left(\frac{1}{\alpha_{1}}\right)=0 \text {, } \\
& \text { II. } r\left(\frac{1}{\alpha_{2}}\right) \neq 0 \text { and } q\left(\frac{1}{\alpha_{1}}\right) \neq 0 \text {, } \\
& \text { III. } r\left(\frac{1}{\alpha_{2}}\right) \neq 0 \text { and } q\left(\frac{1}{\alpha_{1}}\right)=0 \text {. }
\end{aligned}
$$

I. In this case $\alpha_{1}$ and $\alpha_{2}$ are algebraic numbers and we can determine their algebraic conjugates $\left\{x_{1}, \ldots, x_{u}\right\}$ and $\left\{y_{1}, \ldots, y_{v}\right\}$ respectively. Let us write equation (13) into the form

$$
1+\delta_{1}^{-1}\left(\alpha_{1}\right) \beta_{1}+\delta_{1}^{-1}\left(\alpha_{2}\right) \beta_{2}=0
$$

by the inverse of $\delta$. Here

$$
\delta_{1}^{-1}\left(\alpha_{1}\right) \in\left\{x_{1}, \ldots, x_{u}\right\} \text { and } \delta^{-1}\left(\alpha_{2}\right) \in\left\{y_{1}, \ldots, y_{v}\right\} .
$$

Therefore there exists a not identically zero solution iff there exists a pair $\left(x_{i}, y_{j}\right)$ such that it is algebraically conjugated to $\vec{\alpha}=\left(\alpha_{1}, \alpha_{2}\right)$ and

$$
1+x_{i} \beta_{1}+y_{j} \beta_{2}=0,
$$


where $x_{i}$ and $y_{j}$ belong to the finite sets of the algebraic conjugates of $\alpha_{1}$ and $\alpha_{2}$, respectively. Recall that if all the outer parameters are algebraic numbers then we have an alternative method to check the existence of the non-zero additive solution by an algorithm due to A. Varga [2].

II. In this case we can determine the possible values of $\delta_{1}\left(\beta_{1}\right)$ and $\delta_{1}\left(\beta_{2}\right)$ as one of the finitely many roots of the polynomials $p_{1}$ and $p_{2}$, respectively:

$$
\delta_{1}\left(\beta_{1}\right) \in\left\{x_{1}, \ldots, x_{u}\right\} \text { and } \delta_{1}\left(\beta_{2}\right) \in\left\{y_{1}, \ldots, y_{v}\right\} .
$$

Therefore there exists a not identically zero solution iff there exists a pair $\left(x_{i}, y_{j}\right)$ such that it is algebraically conjugated to $\vec{\beta}=\left(\beta_{1}, \beta_{2}\right)$ and

$$
1+\alpha_{1} x_{i}+\alpha_{2} y_{j}=0
$$

where $x_{i}$ and $y_{j}$ belong to the finite sets of the roots of the polynomials $p_{1}$ and $p_{2}$, respectively.

III. In this case we can determine the possible values of $\delta_{1}\left(\beta_{1}\right)$ as one of the finitely many roots of the polynomial $p_{1}: \delta_{1}\left(\beta_{1}\right) \in\left\{x_{1}, \ldots, x_{u}\right\}$. This means that $\delta_{1}\left(\beta_{2}\right)$ can be explicitly expressed from equation (13) in terms of $x_{i}$ (cf. the case of $n=2$ ). Therefore there exists a not identically zero solution iff there exists a root $x_{i}$ such that

$$
\left(\beta_{1}, \beta_{2}\right) \text { and }\left(x_{i},-\frac{1+\alpha_{1} x_{i}}{\alpha_{2}}\right)
$$

are algebraically conjugated. The case of $r\left(\frac{1}{\alpha_{2}}\right)=0$ and $q\left(\frac{1}{\alpha_{1}}\right) \neq 0$ is similar.

\section{An observation about the $A_{1}$ term in case of $n \geq 3$}

The following theorem is a generalization of Theorem 3.1 and 3.2 in [2].

Theorem 3.1. Suppose that $n \geq 3$, the parameters $\beta_{1}, \ldots, \beta_{s}$ are algebraic numbers and $\beta_{s+1}, \ldots, \beta_{n-1}$ are algebraically independent over $\mathbb{Q}$, where $1 \leq$ $s \leq n-1$. If the parameters $\alpha_{1}, \ldots, \alpha_{n-1}$ are algebraic numbers, then the only additive solution of (3) is the identically zero function. 
Proof. If $A_{1}$ is a nonzero additive solution of (3) then by Theorem 1.5 there exists an injective homomorphism $\delta_{1}: \mathbb{Q}\left(\beta_{1}, \ldots, \beta_{n-1}\right) \rightarrow \mathbb{C}$ such that

$$
1+\sum_{i=1}^{n-1} \alpha_{i} \delta_{1}\left(\beta_{i}\right)=0 .
$$

Let us assume that every $\alpha_{i}$ is algebraic. Since $\beta_{1}, \ldots, \beta_{s}$ are algebraic numbers, the algebraic independence of $\beta_{s+1}, \ldots, \beta_{n-1}(s<n-1)$ implies that $\beta_{n-1}$ is transcendental over the algebraic closure of $\mathbb{Q}\left(\beta_{1}, \ldots, \beta_{n-2}\right)$. The exact statement and the proof can be found in [6]. Since any injective homomorphism $\delta_{1}: \mathbb{Q}\left(\beta_{1}, \ldots, \beta_{n-1}\right) \rightarrow \mathbb{C}$ can be extended to an automorphism $\delta_{1}: \mathbb{C} \rightarrow \mathbb{C}$ we can reformulate the previous equation as

$$
1+\sum_{i=1}^{n-1} \delta_{1}^{-1}\left(\alpha_{i}\right) \beta_{i}=0 .
$$

Therefore $\beta_{n-1}$ can be expressed as

$$
\beta_{n-1}=-\frac{1+\sum_{i=1}^{n-2} \delta_{1}^{-1}\left(\alpha_{i}\right) \beta_{i}}{\delta_{1}^{-1}\left(\alpha_{n-1}\right)} .
$$

Since $\delta_{1}^{-1}(\alpha)$ is algebraic, if $\alpha$ is algebraic, the right hand side belongs to the algebraic closure of $\mathbb{Q}\left(\beta_{1}, \ldots, \beta_{n-2}\right)$. This is a contradiction.

The role of the parameters can be changed in the theorem, the proof is similar.

\section{References}

[1] Z. Daróczy, Notwendige und hinreichende Bedingungen fr die Existenz von nichtkonstanten Lsungen linearer Funktionalgleichungen, Acta Sci. Math. Szeged, 22 (1961), 31-41.

[2] A. Varga, On additive solutions of a linear equation, Acta Math Hungar., 128 (1-2), 2010, 15-25.

[3] A. Varga and Cs. Vincze, On Daróczy's problem for additive functions, Publ. Math. Debrecen, Vol. 75 (1-2), 2009, 299-310. 
[4] A. Varga and Cs. Vincze, On a functional equation containing weighted arithmetic means, International Series of Numerical Mathematics, Vol.157, 2009, 305-315

[5] Zs. Páles, Extension theorems for functional equations with bisymmetric operators, Aequat. Math. 63 (2002), 266-291.

[6] M. Kuczma, An Introduction to the Theory of Functional Equations and Inequalities, Prace Naukowe Universitetu Śląskiego w Katowicach Vol. CDLXXXIX (Państwowe Wydawnictwo Naukowe - Universitet Śląski, Warszawa-Kraków-Katowice, 1985).

[7] L. Székelyhidi, On a class of linear functional equations, Publ. Math. (Debrecen) 29 (1982), 19-28.

[8] M. Laczkovich and G. Székelyhidi, Harmonic Analysis on discrete Abelian groups, Proc. Amer. Math. Soc. 133 (2005), 1581-1586.

[9] G. Kiss and A. Varga, Existence of nontrivial solutions of linear functional equations, accepted for publication in Aequat. Math.

[10] M. Laczkovich and G. Kiss, Linear functional equations, differential operators and spectral synthesis, accepted for publication in Aequat. Math.

[11] M. Laczkovich and G. Kiss, Non-constant solutions of linear functional equations, 49th Int. Symp. on Functional equation, Graz (Austria), June 19-26, 2011, http://www.unigraz.at/jens.schwaiger/ISFE49/talks/saturday/Laczkovich.pdf.

Cs. Vincze

Department of Geometry, Institute of Mathematics

Faculty of Science and Technology

University of Debrecen

4010 Debrecen, Hungary P.0. Box 12.

e-mail: csvincze@science.unideb.hu

A. Varga

Faculty of Engineering, University of Debrecen

4010, Debrecen, Hungary P.0. Box 12.

e-mail: vargaa@eng.unideb.hu 
G. Kiss

Department of Stochastics, Faculty of Natural Sciences Budapest University of Technology and Economics and

MTA-BME Stochastics Research Group (04118)

Budapest, Müegyetem rkp. 3.

1111 Hungary

e-mail: kigergo57@gmail.com 